\title{
RECUPERAÇÃO DE COMPOSTOS BIOATIVOS A PARTIR DO BAGAÇO DE UVA ${ }^{1}$
}

\author{
ANA PAULA GIL GRUZ², CARLA GUIMARÃES SILVA E SOUSA ${ }^{3}$ \\ ALEXANDRE GUEDES TORRES ${ }^{4}$, SUELY PEREIRA FREITAS ${ }^{5}$ \\ LOURDES MARIA CORREA CABRAL ${ }^{6}$
}

RESUMO - As indústrias de frutas destacam-se pelo volume de resíduo gerado e, principalmente, pela composição dos mesmos. O Brasil vem-se consolidando como um importante produtor mundial de suco de uva, gerando grande quantidade de resíduos diversos, como o bagaço, que corresponde a aproximadamente $20 \%$ da fruta processada. O bagaço constitui-se de casca e semente, e apresenta uma composição rica e heterogênea em compostos fenólicos (flavonoides e não flavonoides), razão pela qual foi empregado como matéria-prima no presente trabalho para a obtenção de um extrato bioativo. As extrações enzimática e etanólica foram avaliadas para a recuperação destes compostos para fins alimentícios. Dentre as condições avaliadas nos dois planejamentos experimentais, a extração hidroetanólica foi mais eficiente na recuperação dos compostos bioativos ( $\mathrm{p}<0,01$ ), cujo extrato apresentou uma capacidade antioxidante de $1.965 \mu \mathrm{mol}$ Trolox por $100 \mathrm{~g}$ de bagaço, $25 \%$ superior à do extrato obtido com a melhor condição enzimática (1.580 $\mu$ mol Trolox por $100 \mathrm{~g}$ de bagaço).

Termos de indexação: reaproveitamento de coprodutos, Vitis sp., compostos fenólicos, antioxidantes, extração etanólica; extração enzimática.

\section{RECOVERY OF BIOACTIVE COMPOUNDS FROM GRAPE POMACE}

ABSTRACT - Fruit industries are highlighted by the residue amount and especially, for its composition. Brazil thereby is consolidating as an important grape juice producer, generating a large amount of diverse residues like grape pomace, which corresponds around $20 \%$ of total fruit processed. Grape pomace is constituted by seeds and peel and presents rich and heterogeneous composition in phenolic compounds (flavonoids and non flavonoids), reason why it was employed as raw material to obtain a bioactive extract in this study. Enzymatic and ethanolic extractions were evaluated to recover these compounds as they are able for application in food products. Between the evaluated conditions in both experimental design, ethanolic extraction was more efficient for the recovery of bioactive compounds $(\mathrm{p}<0,01)$, which extract presented an antioxidant activity of $1.965 \mu \mathrm{mol}$ Trolox per $100 \mathrm{~g}$ of grape pomace, $25 \%$ higher than the extract obtained in the better enzymatic extraction $(1.580 \mu \mathrm{mol}$ Trolox per $100 \mathrm{~g}$ of grape pomace).

Index terms: by-products reuse, Vitis sp, phenolic compounds, antioxidants, ethanolic extraction, enzymatic extraction.

\footnotetext{
'(Trabalho 200-13). Recebido em: 20-05-2013. Aceito para publicação em: 28-10-2013.

${ }^{2}$ UFRJ - Doutoranda em Ciências de Alimentos (PGCAL) - Laboratório de Bioquímica Nutricional e de Alimentos - Instituto de Química/UFRJ -

-Av. Athos da Silveira Ramos, 149 - CT Bloco A, sala 528A, Ilha Fundão, 21941-909 - Rio de Janeiro, RJ - Brasil. E-mail: *ana_gil@ uol.com.br

${ }^{3}$ UFRRJ - graduanda em Engenharia Química - DEQ - BR 465, km 7, 23890-000 - Seropédica/RJ- Brasil. E-mail: carlagss29@ yahoo.com.br

${ }^{4}$ UFRJ - Prof. Dr. Departamento de Bioquímica - Laboratório de Bioquímica Nutricional e de Alimentos - Instituto de Química/UFRJ - Av. Athos da Silveira Ramos, 149 - CT Bloco A, sala 528A, Ilha Fundão, 21941-909 - Rio de Janeiro, RJ - Brasil. E-mail: torres@iq.ufrj.br ${ }^{5}$ UFRJ- Prof ${ }^{a}$. Dr ${ }^{a}$. Departamento de Engenharia Química - Escola de Química/UFRJ - Av. Athos da Silveira Ramos, 149 - CT Bloco E, Sala 211, Ilha Fundão, 21941-909 - Rio de Janeiro, RJ - Brasil. E-mail: freitasp@eq.ufrj.br

${ }^{6}$ EMBRAPA Agroindústria de Alimentos - Pesquisadora - Av. das Américas, 29501, 23020-470 Rio de Janeiro/RJ, Brasil. E-mail: lourdes.cabral@embrapa.br
} 


\section{INTRODUÇÃO}

A produção de alimentos vem sendo redirecionada para uma produção limpa, com o objetivo de minimizar a geração de resíduos e maximizar o aproveitamento dos recursos empregados. O aproveitamento de resíduos da indústria de alimentos, através da implantação de novos processos integrados, permite aliar a produtividade com a eficiência ambiental (ARVANITOYANNS; VARZAKAS, 2008). As indústrias de alimentos, em especial as processadoras de frutas e hortaliças, geram elevados volumes de subprodutos e resíduos potencialmente ricos em substâncias de alto valor nutricional e funcional (KOSSEVA, 2009). Para minimizar impactos ambientais, esses resíduos têm sido destinados para adubação de terras e complementação de ração animal. A uva é uma das frutas mais cultivadas no mundo e aproximadamente $80 \%$ da produção mundial destina-se à fabricação de vinhos e derivados, gerando diversos resíduos, especialmente, engaço, borra e bagaço. No Brasil, a distribuição da safra apresenta um perfil diferenciado, com pouco menos de $50 \%$ destinado ao processamento, sendo o suco de uva (integral e concentrado) o produto de maior destaque e em plena expansão (MELLO, 2012). O bagaço, na vitinicultura, representa um problema ambiental em função de sua elevada quantidade, de aproximadamente $20 \%$ do volume total processado, gerado em curto intervalo de tempo, com características poluentes (BUSTAMANTE et al., 2008).

O bagaço apresenta teores relativamente elevados de compostos fenólicos com ação fitotóxica e antibacteriana, que dificultam seu descarte ou a utilização direta como adubo ou ração animal (BUSTAMANTE et al., 2007). No entanto, estes mesmos compostos apresentam bioatividade em humanos e vêm-se destacando por sua ação específica em prevenir doenças crônicas graves, tais como doenças cardiovasculares e câncer (LEIFERT; ABEYWARDENA, 2008; PETTI; SCULLY, 2009). Os compostos fenólicos compreendem, simultaneamente, estruturas simples, tais como os ácidos fenólicos, e outras mais complexas, tais como os flavonoides, que apresentam distribuição não homogênea entre os vegetais (NAZCK; SHAHIDHI, 2004). A distribuição de compostos fenólicos na uva é heterogênea quanto aos tecidos do fruto e quanto às classes de compostos. A uva apresenta teores fenólicos totais de 5-8\% nas sementes, $1-4 \%$ no engaço e 1-2\% na casca (SHRIKHANDE, 2000). Além disso, nas cascas, encontram-se flavonoides, tais como antocianinas, flavonóis, flavonóis e proantocianidinas, e não flavonoides, tais como ácidos fenólicos e estilbenos (MAZZA, 1995; MAZZA et al., 1999; MONTEALEGRE et al., 2006; PERESTRELO et al., 2012). Por outro lado, nas sementes, ocorrem predominantemente os taninos condensados.

A recuperação dos compostos fenólicos a partir do bagaço de uva vem sendo investigada, especialmente pela aplicação de diversas tecnologias envolvendo a extração sólido:líquido auxiliada por micro-ondas, pulsos elétricos, alta pressão hidrostática (CORRALES et al. 2008), extração com fluido supercrítico (PALENZUELA et al., 2004) e/ou $\beta$-ciclodextrinas (RATNASOORIYA; RUPASINGHE, 2012). A aplicação potencial dos extratos é o principal fator a ser considerado na escolha das técnicas de extração de componentes bioativos de resíduos da vitivinicultura. Em geral, soluções hidroalcoólicas ou água sulfurada aquecida são os solventes mais utilizados na indústria de alimentos (JU; HOWARD, 2005; MAZZA, 1995). A aplicação de preparações enzimáticas vem sendo investigada como alternativa tecnológica mais sustentável para a obtenção de extratos bioativos (MUÑOZ et al., 2004) de interesse da indústria de alimentos.

Alguns extratos naturais têm sido empregados em formulações alimentícias, tais como os de antioxidantes naturais, com vistas à substituição total ou parcial de aditivos sintéticos potencialmente tóxicos. Devido à pluralidade das espécies químicas de componentes bioativos naturais, tais como os fenólicos, observam-se diferentes mecanismos de ação e grande potencial para sinergismos, que podem aumentar sua ação nos sistemas a serem empregados, em comparação com o uso de substâncias isoladas (DOSSIÊ ANTIOXIDANTES, 2009).

O objetivo do presente trabalho foi comparar dois processos tecnológicos na recuperação de compostos bioativos do bagaço da uva com vistas à aplicação em alimentos. Foram avaliadas as extrações enzimática e hidroetanólica, ambas em escala laboratorial.

\section{MATERIAIS E MÉTODOS}

\section{Material}

A uva rosada, normalmente usada para a produção de sucos, foi adquirida no comércio local. O bagaço de uva utilizado nos experimentos foi obtido por prensagem hidráulica de $7,8 \mathrm{~kg}$ das uvas desengaçadas manualmente, sanitizadas e acondicionadas em sacos com tela de náilon 
(diâmetro de $150 \mu \mathrm{m}$ ).

Enzima: A preparação enzimática empregada possui atividade de pectinase e hemicelulase proveniente dos microrganismos Aspergillus niger e Trichoderma reseei, semelhante à melhor preparação testada por Muñoz et al. (2004) para a recuperação de antocianinas do bagaço de uva fermentado.

\section{Metodologia}

Extração: - Os processos de extração foram avaliados a partir de dois planejamentos fatoriais $2^{4}$ com triplicata no ponto central. Os fatores avaliados nos dois planejamentos experimentais foram o $\mathrm{pH}$, a razão solvente/substrato e a temperatura. Adicionalmente, foram avaliados o teor de etanol e a concentração de enzimas para as extrações hidroetanólica e enzimática, respectivamente, cujos níveis estão apresentados na Tabela 1 . As variáveis de resposta selecionadas foram o teor de compostos fenólicos totais, teor de antocianinas totais e monoméricas, bem como a capacidade antioxidante.

As misturas foram preparadas em erlenmeyers, e os experimentos de extração, conduzidos em shaker com temperatura controlada e agitação de $60 \mathrm{rpm}$, por 120 minutos. Os sólidos em suspensão foram reduzidos através da filtração em funil de Büchner sem papel de filtro, e os extratos obtidos foram congelados a $-20^{\circ} \mathrm{C}$ até análise.

Determinação dos compostos fenólicos totais (FT): - Os compostos fenólicos totais foram quantificados como descrito por Singleton e Rossi (1965), de acordo com as modificações de Georgé et al. (2005), usando-se ácido gálico como padrão analítico. Os extratos foram diluídos em acetona a $70 \%$ e após 30 minutos de agitação magnética foram filtrados em papel de filtro de filtração rápida. Uma alíquota de $0,5 \mathrm{~mL}$ do filtrado diluído em água destilada ou de solução de ácido gálico foi transferida para um tubo de ensaio. A seguir, foram adicionados $2,5 \mathrm{~mL}$ de reativo de Folin-Ciocalteau a $10 \%$ em água e $2,0 \mathrm{~mL}$ de carbonato de sódio a 7,5\% em água. Os tubos foram agitados, transferidos para um banho-maria a $50^{\circ} \mathrm{C}$ e, após 15 minutos de reação, os mesmos foram conservados em um banho de gelo. A absorvância das soluções à temperatura ambiente foi medida em espectrofotômetro (Bioespectro modelo SP210) a $760 \mathrm{~nm}$. A quantificação baseou-se na curva de calibração de ácido gálico (0 a $80 \mathrm{mg} / \mathrm{L}$ ), e os resultados foram expressos como ácido gálico equivalente por $100 \mathrm{~g}$ de extrato $(\mathrm{mg} / 100 \mathrm{~g})$.

Determinação das antocianinas totais (AT) e monoméricas (AM): - As antocianinas totais e monoméricas foram quantificadas pelo método do $\mathrm{pH}$ diferencial (GIUSTI; WROLSTAD, 2001), utilizando a cianidina-3-glucosídeo como referência e os dados de coeficiente de extinção molar $(\varepsilon)$ da literatura $\left(26.900 \mathrm{M}^{-1} \mathrm{~cm}^{-1}\right)$ e massa molecular (PM) de 449,2 g.gmol ${ }^{-1}$. Os extratos obtidos foram diluídos em tampão pH 1,0 e pH 4,5, separadamente, e após 30 minutos de estabilização, as absorbâncias das soluções foram lidas a 510 e $700 \mathrm{~nm}$.

A quantificação das antocianinas totais (AT) foi realizada de acordo com a equação 1 , enquanto as antocianinas monoméricas (AM) foram quantificadas de acordo com a equação 2 . Os resultados foram expressos em mg cianidina-3-glucosídeo por $100 \mathrm{~g}$ de extrato.

$$
\begin{aligned}
& A \mathrm{~T}=\left[\left(\frac{\left(\left(\mathrm{Abs}_{510}-\mathrm{Abs}_{700}\right)_{\mathrm{pH} \mathrm{1.0}} \times 10^{3} \times \mathrm{PM} \times \mathrm{fd}\right)}{\varepsilon}\right) \times 100\right] \\
& A \mathrm{M}=\mathrm{AT}-\left[\left(\frac{\left.\left(\mathrm{Abs}_{510}-\mathrm{Abs}_{700}\right)_{\mathrm{pH} 4.5} \times 10^{3} \times \mathrm{PM} \times \mathrm{fd}\right)}{\varepsilon}\right) \times 100\right]
\end{aligned}
$$

Determinação da capacidade antioxidante: -Os extratos obtidos no item 2.1 foram submetidos à extração analítica prévia, de acordo com Rufino et al. (2007), e a determinação da capacidade antioxidante foi avaliada pelo método do ABTS expresso em equivalentes de Trolox (RE et al., 1999). O radical $\mathrm{ABTS}^{+}$foi obtido quimicamente por oxidação estequiométrica do ABTS por persulfato de potássio. Alíquotas das soluções aquosas de ABTS $7 \mathrm{mM}$ e Persulfato de Potássio $140 \mathrm{mM}$ foram transferidas para um frasco âmbar na razão estequiométrica de 1:0,5 (mol:mol). Para a completa reação e estabilização do radical, o frasco permaneceu ao abrigo da luz, à temperatura ambiente, por 14 horas, sendo esta a solução radicalar concentrada. A solução concentrada $\mathrm{ABTS}^{+}$foi diluída em etanol a $95 \%$ até se atingir uma absorbância a $734 \mathrm{~nm}$ de $0,700 \pm 0,02$. Soluções de Trolox ${ }^{\circledR}(100 ; 500 ; 1.000 ; 1.500$ e 2.000 $\mu \mathrm{M}$ em etanol a $95 \%$ ) e os extratos foram transferidos para tubos de ensaio onde reagiram por 6 minutos com a solução diluída de $\mathrm{ABTS}^{+}{ }^{+}$, na proporção de $1: 10$, à temperatura ambiente e ao abrigo da luz. A cada triplicata analítica, um branco com etanol a 95\% foi lido, e a diferença entre as absorbâncias do branco e as amostras ou o padrão foi utilizada para os cálculos. Os resultados obtidos foram expressos em $\mu \mathrm{mol}$ Trolox/g extrato.

Análise estatística:- Os resultados analíticos dos extratos foram calculados em termos de rendimento, referente a $100 \mathrm{~g}$ de bagaço. Os resultados foram expressos como média e desvio-padrão com, ao menos, três replicatas analíticas. Os resultados do planejamento experimental (fatorial $2^{4} \mathrm{com}$ triplicata 
no ponto central) e as médias analíticas foram avaliados, empregando-se os softwares Statistica 7.0 e software XLSTAT 7.5. Os fatores avaliados foram considerados estatisticamente significativos para valores de $p<0,05$ e marginalmente significativos para $0,05 \leq \mathrm{p} \leq 0,10$.

\section{RESULTADOS E DISCUSSÃO}

Extração Enzimática: - Todas as variáveis investigadas apresentaram distribuição normal, de acordo com os testes de Shapiro-Wilk e KolmogorovSmirnov.

A quantidade de enzima adicionada teve por base dados reportados na literatura para extração de suco de amora-preta (SANTIAGO, 2010). Na faixa analisada, os resultados obtidos para a extração enzimática indicaram a influência positiva dos fatores razão solvente/substrato e temperatura para todas as variáveis de resposta. Como esperado, o aumento da razão solvente/carga favoreceu a transferência de massa da matriz sólida para a solução extratora, por aumentar o gradiente de potencial químico. Neste caso o solvente, água pura, foi capaz de extrair os compostos mais polares e fracamente ligados na estrutura da matriz.

O aumento da temperatura também contribuiu de forma positiva para a recuperação dos compostos bioativos, muito provavelmente pelas características da preparação enzimática empregada, cuja condição ótima é alcançada a $50^{\circ} \mathrm{C}$ e pH 3.5, porém com uma faixa de atividade entre 10 a $50{ }^{\circ} \mathrm{C}$ e pH de 3,0 a 5,5 . Como pode ser observado no gráfico de valores marginais (Figura 1), o aumento da temperatura de 30 para $50{ }^{\circ} \mathrm{C}$ em $\mathrm{pH} 2.0$ promoveu acréscimo de $47 \%$ na atividade antioxidante do extrato, passando de 644 para $946 \mu \mathrm{mol} \mathrm{TE} / 100 \mathrm{~g}$. Em pH 4,0, o efeito da temperatura foi maior ainda, uma vez que o extrato obtido a $50^{\circ} \mathrm{C}$ apresentou atividade antioxidante $83 \%$ superior ao obtido a $30^{\circ} \mathrm{C}$ (Figura 1).

A redução do $\mathrm{pH}$ da solução promoveu amento das antocianinas no extrato, muito provavelmente por estabilizar o núcleo flavílio. Porém, para a capacidade antioxidante, o pH mostrou-se marginalmente significativo $(\mathrm{p} \leq 0,10)$. Neste caso, o aumento do $\mathrm{pH}$ da solução aumentou a capacidade antioxidante do extrato, que pode ser decorrente da maior estabilidade da preparação enzimática e, consequentemente, maior extração de compostos bioativos e não somente os compostos fenólicos determinados neste trabalho.

A capacidade antioxidante é favorecida com o aumento da temperatura e da razão solvente/ substrato (Figura 2); no entanto, o fator de interação entre estas duas variáveis independentes foi apenas marginalmente significativo $(\mathrm{p} \leq 0,10)$. Já para a extração dos compostos fenólicos, o fator de interação entre a temperatura e a razão solvente/ substrato foi significativo (Figura 3).

Embora os maiores rendimentos de extração dos compostos fenólicos totais $(286,1 \pm 1,8 \mathrm{mg} / 100 \mathrm{~g})$ e antocianinas $(32,8 \pm 0,6 \mathrm{mg} / 100 \mathrm{~g})$ tenham ocorrido nas condições do ensaio $10-\mathrm{pH} 2,0 ; 50{ }^{\circ} \mathrm{C}$; razão solvente/substrato 3:1; e $630 \mathrm{UI} / 100 \mathrm{~g}$ bagaço (Tabela 2) - a capacidade antioxidante do extrato foi maior em pH 4,0 (ensaios 12 e 16). Como a curvatura não foi significativa estatisticamente, pode-se inferir que, dentre as condições avaliadas, as dos ensaios 12 e 16 foram as mais eficientes quando se trata da capacidade antioxidante, e não apresentaram diferença significativa entre si $(\mathrm{p}>0,1)$. As condições destes ensaios foram: $\mathrm{pH} 4,0(+1)$, razão solvente/ substrato de $3: 1(+1)$, temperatura de $50{ }^{\circ} \mathrm{C}(+1) \mathrm{em}$ ambos os níveis de enzima ( 210 e $630 \mathrm{UI} / 100 \mathrm{~g}$ de bagaço), respectivamente, sugerindo um excesso da preparação enzimática no meio extrator.

\section{Extração Hidroetanólica:}

Os valores de rendimento em capacidade antioxidante variaram de 600 a $2.000 \mu$ mol Trolox equivalente por $100 \mathrm{~g}$ de bagaço na extração hidroetanólica (Tabela 3 ), um pouco acima da faixa de 440 a $1.600 \mu \mathrm{mol}$ Trolox equivalente por 100 $\mathrm{g}$ de bagaço observada para a extração enzimática (Tabela 2).

Assim como no processo enzimático, a razão solvente/carga e a temperatura foram os parâmetros estatisticamente significativos para todas as variáveis de resposta avaliadas. A influência destes fatores foi positiva, porém verificou-se que, para as antocianinas, a razão solvente/carga foi mais relevante que a temperatura (Figura 4), enquanto para os fenólicos totais e atividade antioxidante ocorreu o inverso, o aumento da temperatura atuou mais fortemente que a razão solvente/substrato (Figuras 5 e 6$)$.

A temperatura teve um efeito positivo no rendimento de extração etanólica, provavelmente devido ao aumento do coeficiente de difusão e da solubilidade das moléculas no solvente, promovidos pelo aumento da temperatura. Spigno et al. (2007) verificaram o mesmo comportamento, sendo a extração dos fenólicos de bagaço de uva a $60^{\circ} \mathrm{C}$ mais eficiente que a conduzida a $45^{\circ} \mathrm{C}$. Contudo, após 20 horas de extração, foram observadas perdas nos rendimentos, em ambas as temperaturas. Embora a maioria dos compostos bioativos recuperados sejam termolábeis, não se verificaram perdas com a temperatura de $50^{\circ} \mathrm{C}$, muito provavelmente por 
o tempo de extração ser de apenas duas horas. Os aumentos no teor de etanol e na temperatura da extração favoreceram o rendimento em atividade antioxidante (Figura 6).

$\mathrm{O}$ aumento do teor de etanol favoreceu a extração dos compostos fenólicos totais, enquanto para as antocianinas o efeito foi oposto (Figura 7). Acredita-se que, embora as antocianinas pertençam à classe dos compostos fenólicos, outras subclasses também são mensuradas pela metodologia de Folin empregada, e, neste caso, a modificação da polaridade pelo aumento do teor de etanol seja mais favorável para a maioria destas subclasses. Para as antocianinas, observa-se que o etanol somente influi negativamente quando em temperatura mais baixa $\left(30{ }^{\circ} \mathrm{C}\right)$, em que a solubilidade destes compostos depende mais fortemente da polaridade por serem pigmentos hidrossolúveis. Entretanto, a $50^{\circ} \mathrm{C}$, acredita-se que efeito da concentração do etanol na solução seja sobreposto pelo aumento do coeficiente de difusão ocasionado pelo aumento da temperatura. Outro fato importante que deve ser considerado é o baixo teor de antocianinas nesta matriz, se comparado ao de outros alimentos, tais como o açaí e outras uvas tintas, que pode ser um fator a contribuir para a maior intensidade das interações mencionadas acima.

$\mathrm{O} \mathrm{pH}$ teve efeito significativo e foi diretamente associado com os teores de antocianinas totais no extrato, assim como observado na extração enzimática, e diferentemente do observado por Valduga et al. (2008). Possivelmente, em pH 4,0, a quantidade de água livre, capaz de solvatar as antocianinas, foi maior do que no $\mathrm{pH}$ 2,0, uma vez que a acidificação se deu por adição de um ácido fraco (ac. cítrico). Adicionalmente, o pH 4,0 é suficientemente ácido para manter a estabilidade do cátion flavílio (FRANCIS, 1982) e, portanto, essa foi a condição mais favorável no presente trabalho. Em contraste, Valduga et al. (2008) observaram melhor extração de antocianinas nos menores valores de $\mathrm{pH}$, provavelmente devido ao uso de um ácido forte (ác. clorídrico) que não provocou o efeito de salting-out comentado acima. É possível que em valores de $\mathrm{pH}<$ 2,0, especialmente pelo uso de um ácido corrosivo, possa ter ocorrido ruptura de estruturas celulares, favorecendo a recuperação das antocianinas. Por outro lado, na extração enzimática, o pH atuou na estabilização do preparado enzimático, favorecendo sua ação.

Amendola et al. (2010) obtiveram um extrato hidroetanólico de bagaço de uva com teor de fenólicos totais de $7,2 \mathrm{~g} / \mathrm{L}$, um teor muito superior a $1,2 \mathrm{~g} / \mathrm{L}$, atingido neste trabalho no experimento 16, muito provavelmente em decorrência não somente da cultivar, mas das condições de processo que, no trabalho citado, envolveram a secagem e a moagem do bagaço, casca e semente. Embora seja de conhecimento que a área de contato do material está diretamente relacionada ao rendimento da extração, no presente trabalho optou-se por utilizar o bagaço com as dimensões iniciais para reduzir o número de operações unitárias do processamento e também o teor de taninos condensados nos extratos, uma vez que estes se encontram majoritariamente nas sementes. Embora os taninos condensados contribuam fortemente para a atividade antioxidante, são responsáveis pela adstringência nos alimentos e reduzem a digestibilidade de proteínas (NAZCK; SHAHIDHI, 2004). Desta forma, poderiam, respectivamente, afetar as propriedades sensoriais e reduzir a qualidade nutricional dos produtos nos quais o extrato fosse empregado ou até limitar seu uso em preparações alimentícias.

Embora, na maioria dos casos, o aumento da temperatura, da razão solvente/carga e do teor de solvente melhore a extração de solutos, as características da matriz e dos compostos de interesse são primordiais para se estabelecer a condição operacional mais favorável do ponto de vista termodinâmico e econômico.

Considerando que a capacidade antioxidante do extrato é a variável de resposta que traduz a condição dos compostos extraídos, pode-se observar que as melhores condições do processo enzimático e etanólico foram as dos ensaios 12 e 16 para ambos. De acordo com a análise de variância e comparação de médias pelo teste de Fisher ( $\mathrm{p}<0,05)$, as extrações hidroetanólicas foram as mais apropriadas para a obtenção de extrato bioativo de bagaço de uva rosada. 
TABELA 1 -Fatores avaliados nos planejamentos e seus respectivos níveis em valores absolutos e codificados.

\begin{tabular}{lccc}
\hline Fatores & Nível inferior $(-1)$ & Ponto Central $(0)$ & Nível superior $(+1)$ \\
\hline Temperatura $\left({ }^{\circ} \mathrm{C}\right)$ & 30 & 40 & 50 \\
pH & 2 & 3 & 4 \\
Razão solvente/substrato $(\mathrm{m} / \mathrm{v})$ & $1: 1$ & $1: 2$ & $1: 3$ \\
Preparação enzimática $(\mathrm{UI} / 100 \mathrm{~g}) *$ & 210 & 420 & 630 \\
Etanol $(\%)$ & 30 & 50 & 70 \\
\hline
\end{tabular}

* Atividade enzimática medida em atividade de poligalacturonase.

TABELA 2 - Matriz experimental da extração enzimática do bagaço de uva e resultados analíticos obtidos.

\begin{tabular}{ccccccccc}
\hline Ensaios & $\mathrm{T}^{\circ} \mathrm{C}$ & $\mathrm{E}$ & $\mathrm{pH}$ & $\mathrm{S}: \mathrm{S}$ & $\mathrm{AA}$ & $\mathrm{FT}$ & $\mathrm{AT}$ & $\mathrm{AM}$ \\
\hline 1 & -1 & -1 & -1 & -1 & $496,7 \pm 31,2$ & $114,5 \pm 0,9$ & $15,0 \pm 0,1$ & $12,7 \pm 0,1$ \\
2 & -1 & -1 & -1 & +1 & $786,3 \pm 9,0$ & $193,9 \pm 5,8$ & $26,5 \pm 0,4$ & $22,6 \pm 0,5$ \\
3 & -1 & -1 & +1 & -1 & $455,7 \pm 18,8$ & $177,4 \pm 10,8$ & $8,2 \pm 0,2$ & $7,0 \pm 0,2$ \\
4 & -1 & -1 & +1 & +1 & $744,7 \pm 13,0$ & $120,9 \pm 12,2$ & $14,0 \pm 0,4$ & $11,8 \pm 0,6$ \\
5 & -1 & +1 & -1 & -1 & $536,1 \pm 31,2$ & $144,8 \pm 0,9$ & $18,3 \pm 0,2$ & $15,6 \pm 0,3$ \\
6 & -1 & +1 & -1 & +1 & $784,9 \pm 44,8$ & $171,0 \pm 3,7$ & $24,1 \pm 0,2$ & $20,5 \pm 0,8$ \\
7 & -1 & +1 & +1 & -1 & $579,4 \pm 18,0$ & $102,2 \pm 8,4$ & $9,6 \pm 0,2$ & $8,3 \pm 0,1$ \\
8 & -1 & +1 & +1 & +1 & $879,5 \pm 17,6$ & $143,1 \pm 15,1$ & $14,7 \pm 0,1$ & $12,2 \pm 0,7$ \\
9 & +1 & -1 & -1 & -1 & $658,7 \pm 30,5$ & $148,0 \pm 9,2$ & $17,8 \pm 0,1$ & $15,2 \pm 0,1$ \\
10 & +1 & -1 & -1 & +1 & $1257,0 \pm 25,8$ & $286,1 \pm 1,8$ & $32,8 \pm 0,6$ & $28,1 \pm 0,7$ \\
11 & +1 & -1 & +1 & -1 & $722,3 \pm 35,4$ & $111,5 \pm 1,6$ & $11,3 \pm 0,2$ & $9,7 \pm 0,2$ \\
$\mathbf{1 2}$ & $+\mathbf{1}$ & $\mathbf{- 1}$ & $+\mathbf{1}$ & $+\mathbf{1}$ & $\mathbf{1 5 6 0 , 2} \pm \mathbf{6 3 , 7}$ & $\mathbf{2 3 5 , 6} \pm 24,7$ & $\mathbf{2 2 , 3} \pm 0,4$ & $\mathbf{1 8 , 9 5} \pm 0,8$ \\
13 & +1 & +1 & -1 & -1 & $823,5 \pm 27,1$ & $204,1 \pm 1,2$ & $20,0 \pm 0,3$ & $17,31 \pm 0,4$ \\
14 & +1 & +1 & -1 & +1 & $1072,1 \pm 23,3$ & $232,3 \pm 17,2$ & $31,0 \pm 0,6$ & $26,31 \pm 0,6$ \\
15 & +1 & +1 & +1 & -1 & $992,0 \pm 28,8$ & $156,2 \pm 10,2$ & $14,2 \pm 0,1$ & $12,2 \pm 0,1$ \\
$\mathbf{1 6}$ & $+\mathbf{1}$ & $+\mathbf{1}$ & $+\mathbf{1}$ & $+\mathbf{1}$ & $\mathbf{1 5 7 9 , 2} \pm \mathbf{5 0 , 0}$ & $\mathbf{2 4 5 , 1} \pm 1,6$ & $\mathbf{2 1 , 1} \pm 0,5$ & $\mathbf{1 7 , 9} \pm 0,6$ \\
17 & 0 & 0 & 0 & 0 & $\mathbf{7 5 0 1 \pm 3 4 , 5}$ & $166,4 \pm 13,6$ & $21,7 \pm 0,8$ & $18,9 \pm 0,8$ \\
18 & 0 & 0 & 0 & 0 & $902,7 \pm 51,3$ & $175,2 \pm 9,5$ & $20,1 \pm 0,3$ & $17,4 \pm 0,2$ \\
19 & 0 & 0 & 0 & 0 & $824,8 \pm 23,0$ & $198,2 \pm 19,21$ & $23,4 \pm 0,2$ & $20,5 \pm 0,2$ \\
\hline
\end{tabular}

$\mathrm{T}^{\circ} \mathrm{C}$ - temperatura em ${ }^{\circ} \mathrm{C} ; \mathrm{S} / \mathrm{S}$ - razão solvente/substrato; $\mathrm{E}$ - enzima em atividade de poligalacturonase; AA - atividade antioxidante expressa em $\mu \mathrm{mol}$ Trolox/100 g de bagaço; FT - fenólicos totais expressos em mg de ácido gálico equivalente por $100 \mathrm{~g}$ de bagaço; AT- antocianinas totais - expressas em mg de cianidina-3-glucosídeo por $100 \mathrm{~g}$ de bagaço, e AM - antocianinas monoméricas expressas em mg de cianidina-3-glucosídeo por 100g de bagaço. 
TABELA 3 -Matriz experimental da extração hidroetanólica do bagaço de uva e resultados analíticos obtidos.

\begin{tabular}{ccccccccc}
\hline Ensaios & ${ }^{\circ} \mathrm{C}$ & Etanol & $\mathrm{pH}$ & $\mathrm{S}: \mathrm{S}$ & $\mathrm{AA}$ & $\mathrm{FT}$ & $\mathrm{AT}$ & $\mathrm{AM}$ \\
\hline 1 & -1 & -1 & -1 & -1 & $890,6 \pm 24,1$ & $125,3 \pm 1,8$ & $16,7 \pm 1,4$ & $14,2 \pm 0,5$ \\
2 & -1 & -1 & -1 & +1 & $751,30 \pm 27,3$ & $176,1 \pm 5,4$ & $20,7 \pm 0,3$ & $19,2 \pm 0,2$ \\
3 & -1 & -1 & +1 & -1 & $719,35 \pm 51,9$ & $114,4 \pm 1,7$ & $15,1 \pm 0,3$ & $12,7 \pm 0,2$ \\
4 & -1 & -1 & +1 & +1 & $999,31 \pm 72,0$ & $139,3 \pm 4,5$ & $27,0 \pm 1,7$ & $25,6 \pm 1,9$ \\
5 & -1 & +1 & -1 & -1 & $639,56 \pm 42,0$ & $144,8 \pm 1,0$ & $14,0 \pm 0,6$ & $11,7 \pm 0,6$ \\
6 & -1 & +1 & -1 & +1 & $934,8 \pm 52,4$ & $196,2 \pm 6,1$ & $19,6 \pm 0,4$ & $15,9 \pm 0,7$ \\
7 & -1 & +1 & +1 & -1 & $1198,70 \pm 20,8$ & $154,8 \pm 11,5$ & $16,7 \pm 0,3$ & $14,6 \pm 0,4$ \\
8 & -1 & +1 & +1 & +1 & $1150,4 \pm 52,7$ & $209,0 \pm 11,03$ & $17,6 \pm 0,5$ & $16,2 \pm 0,6$ \\
9 & +1 & -1 & -1 & -1 & $906,8 \pm 36,1$ & $188,6 \pm 8,3$ & $18,2 \pm 0,1$ & $15,4 \pm 0,4$ \\
10 & +1 & -1 & -1 & +1 & $1393,6 \pm 60,3$ & $301,0 \pm 15,4$ & $26,6 \pm 0,7$ & $22,3 \pm 0,6$ \\
11 & +1 & -1 & +1 & -1 & $1153,4 \pm 14,5$ & $183,3 \pm 14,8$ & $21,3 \pm 0,2$ & $18,1 \pm 0,2$ \\
$\mathbf{1 2}$ & $+\mathbf{1}$ & $\mathbf{- 1}$ & $+\mathbf{1}$ & $+\mathbf{1}$ & $\mathbf{1 9 6 5 , 4} \pm 68,6$ & $279,6 \pm 7,5$ & $28,9 \pm 0,3$ & $24,6 \pm 0,4$ \\
13 & +1 & +1 & -1 & -1 & $1324,1 \pm 80,7$ & $263,0 \pm 4,1$ & $19,6 \pm 0,3$ & $16,2 \pm 0,3$ \\
14 & +1 & +1 & -1 & +1 & $1741,3 \pm 124,6$ & $393,0 \pm 3,1$ & $30,1 \pm 0,6$ & $24,6 \pm 0,6$ \\
15 & +1 & +1 & +1 & -1 & $1397,3 \pm 91,7$ & $207,5 \pm 6,9$ & $17,3 \pm 0,3$ & $14,6 \pm 0,4$ \\
$\mathbf{1 6}$ & $+\mathbf{1}$ & $+\mathbf{1}$ & $+\mathbf{1}$ & $+\mathbf{1}$ & $\mathbf{1 8 2 6 , 7 \pm 1 1 4 , 2}$ & $347,1 \pm 9,0$ & $28,3 \pm 0,3$ & $24,6 \pm 0,6$ \\
17 & 0 & 0 & 0 & 0 & $1181,5 \pm 74,8$ & $233,4 \pm 12,5$ & $22,7 \pm 0,2$ & $19,9 \pm 0,2$ \\
18 & 0 & 0 & 0 & 0 & $1448,0 \pm 78,7$ & $256,9 \pm 6,8$ & $21,9 \pm 0,6$ & $18,6 \pm 0,5$ \\
19 & 0 & 0 & 0 & 0 & $1311,3 \pm 10,0$ & $217,2 \pm 15,2$ & $22,1 \pm 0,3$ & $18,8 \pm 0,4$ \\
\hline
\end{tabular}

$\mathrm{T}^{\circ} \mathrm{C}$ - temperatura em ${ }^{\circ} \mathrm{C} ; \mathrm{S} / \mathrm{S}$ - razão solvente/substrato; E - \% de etanol na solução extratora; $\mathrm{AA}$ - atividade antioxidante expressa em $\mu \mathrm{mol}$ Trolox/100 g de bagaço; FT - fenólicos totais expressos em mg de ácido gálico equivalente por $100 \mathrm{~g}$ de bagaço; AT- antocianinas totais - expressas em mg de cianidina-3-glucosídeo por $100 \mathrm{~g}$ de bagaço, e AM - antocianinas monoméricas expressas em mg de cianidina-3-glucosídeo por $100 \mathrm{~g}$ de bagaço.

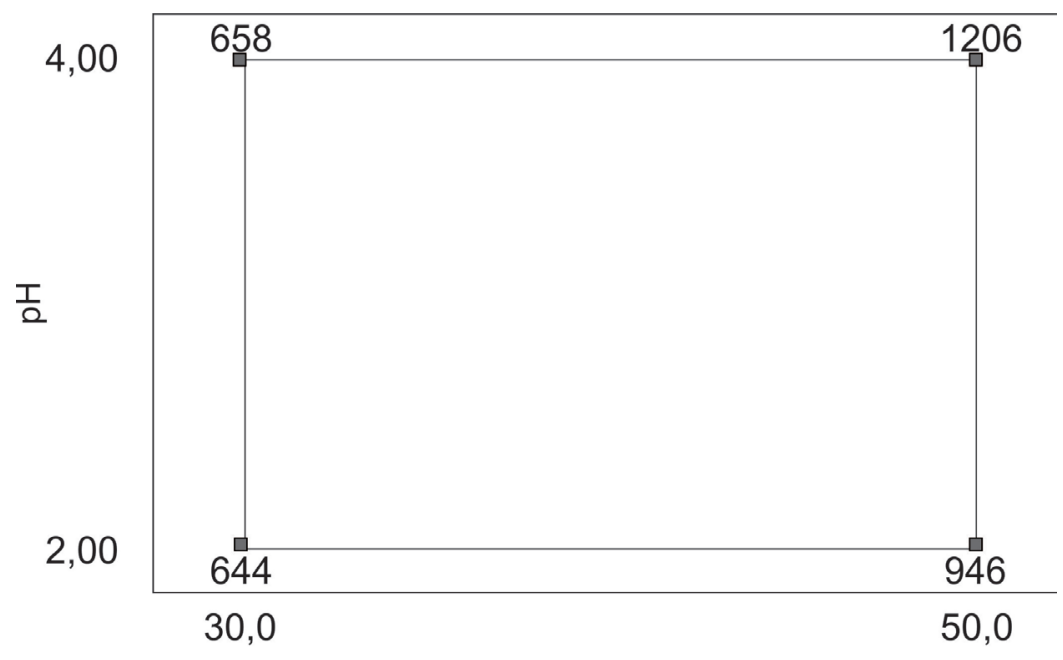

Temperatura

FIGURA 1 -Valores marginais da atividade antioxidante em função do pH e da temperatura. 
(4) Razão solvente:substrato

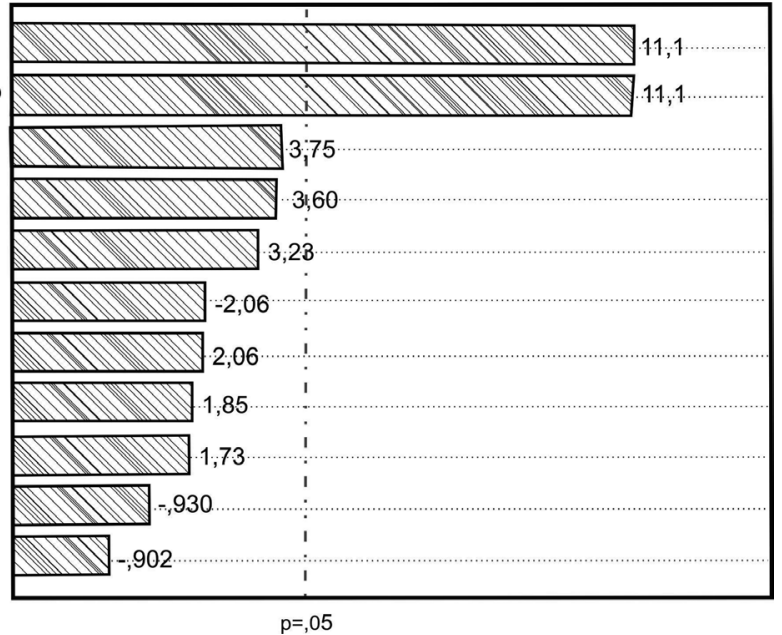

FIGURA 2- Diagrama de Pareto para avaliar o efeito dos fatores investigados sobre a atividade antioxidante nos extratos de bagaço de uva.

(4) Razão solvente substrato

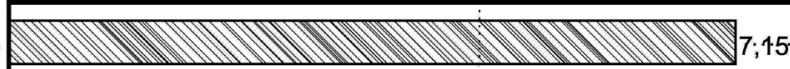

(1) Temperatura

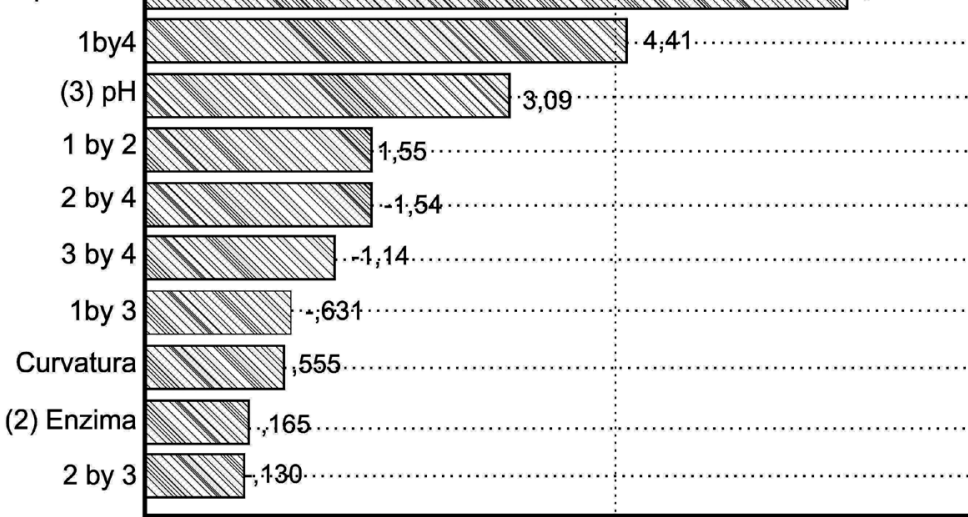

FIGURA 3 -Diagrama de Pareto para avaliar os efeitos dos fatores investigados sobre os compostos fenólicos totais nos extratos de bagaço de uva.

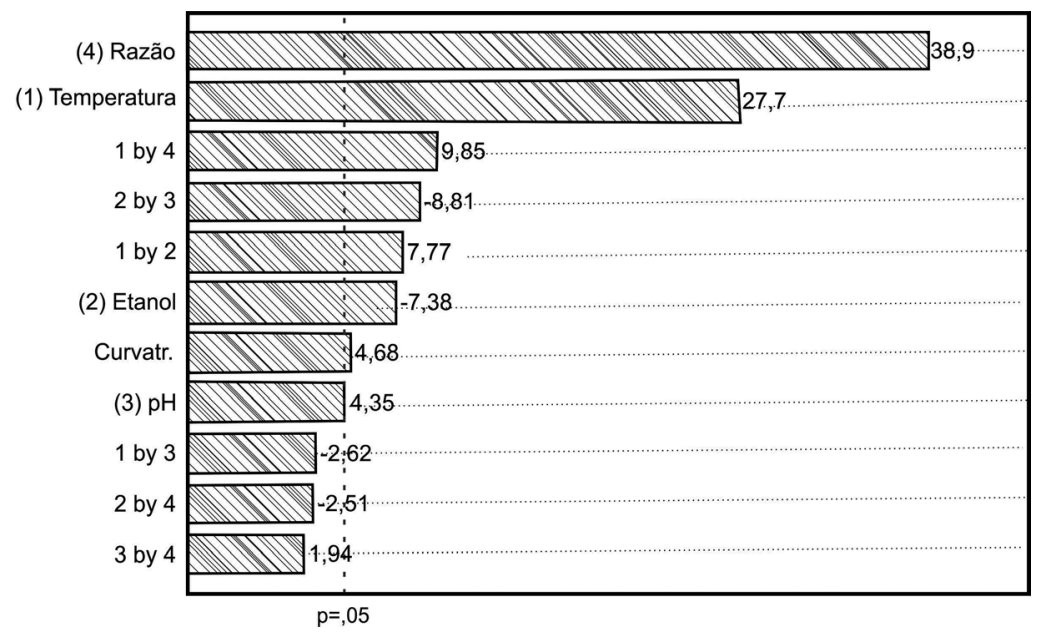

FIGURA 4 -Diagrama de Pareto para avaliar o efeito dos fatores investigados sobre as antocianinastotais presentes nos extratos de bagaço de uva. 


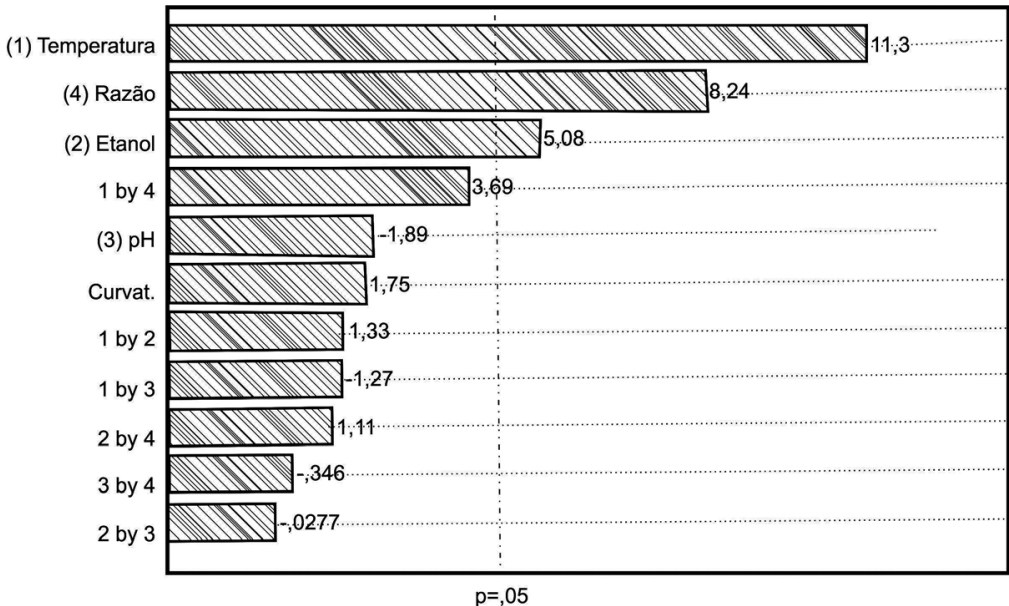

FIGURA 5 - Diagrama de Pareto para avaliar o efeito dos fatores investigados sobre os compostos fenólicos totais nos extratos de bagaço de uva.

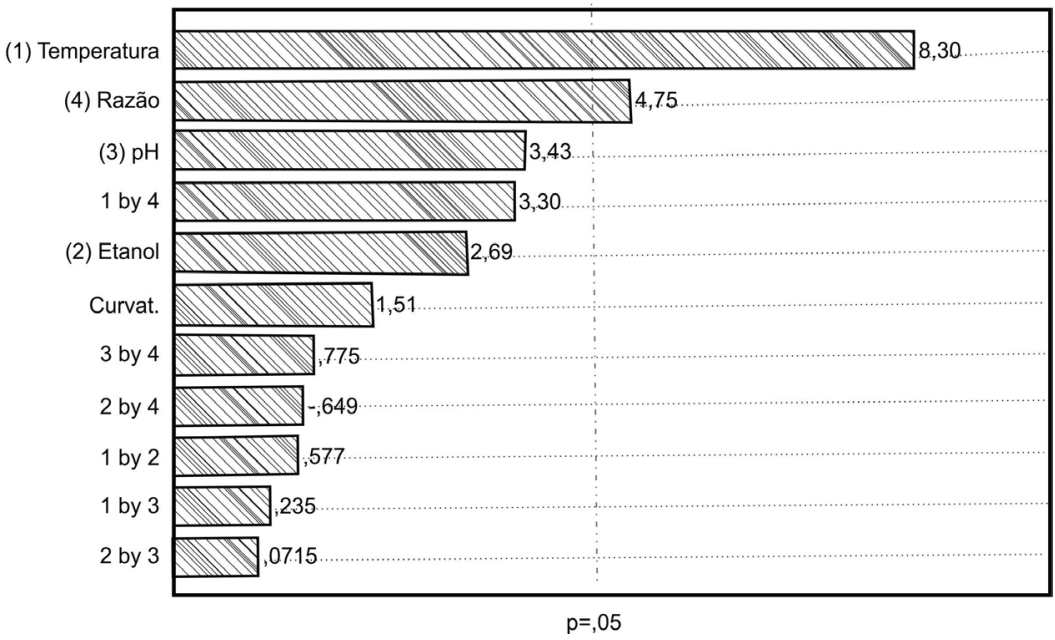

FIGURA 6 - Diagrama de Pareto para avaliar o efeito dos fatores investigados sobre a atividade antioxidante nos extratos de bagaço de uva.
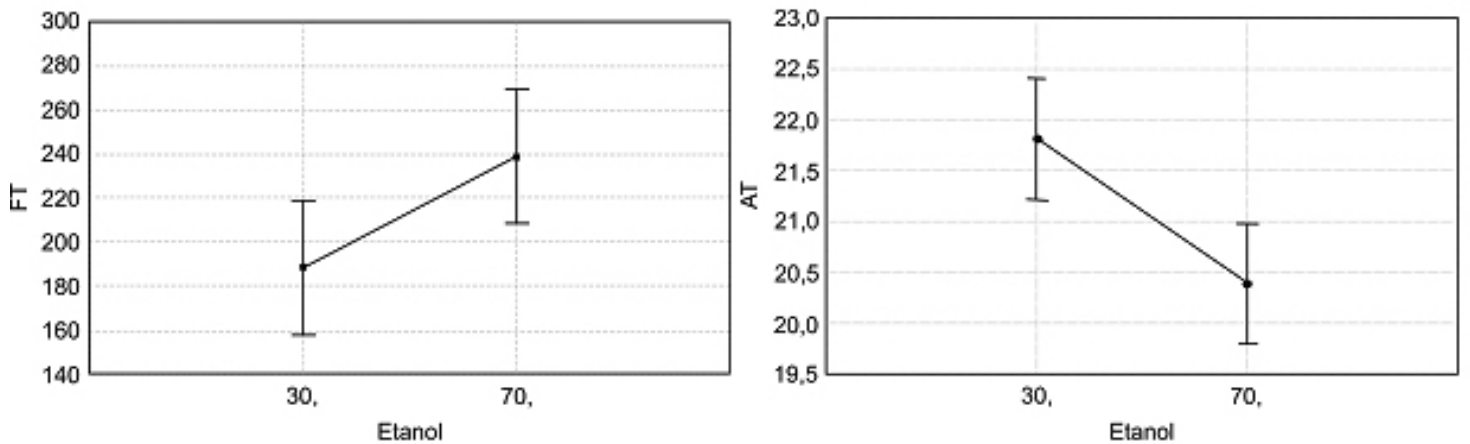

FIGURA 7- Valores marginais de fenólicos totais (esquerda) e antocianinas totais (direita) nos extratos de bagaço de uva, em função do teor de etanol na solução extratora.s respectivos níveis em valores absolutos. 


\section{CONCLUSÃO}

Os fatores razão solvente/substrato e a temperatura influenciaram positivamente na capacidade antioxidante dos extratos obtidos via extração aquosa enzimática e etanólica. De acordo com os resultados obtidos, recomenda-se a extração com etanol:água $(30: 70, v / v)$ conduzida a $50{ }^{\circ} \mathrm{C}, \operatorname{com} \mathrm{pH} 4,0$, e razão solvente/substrato $3: 1$, como a condição termodinâmica mais favorável para a obtenção de extrato bioativo do bagaço de uva rosada.

\section{AGRADECIMENTO}

CAPES, CNPQ e FAPERJ, pelo apoio financeiro; a Selma Terzi, pelas análises de atividade enzimática da preparação enzimática comercial utilizada, e a Luiz Fernando Menezes da Silva, pela elaboração das imagens.

\section{REFERÊNCIAS}

AMENDOLA, D.;DE FAVERI,M.D.; SPIGNO. G. Grape marc phenolics: Extraction kinetics, quality and stability of extracts. Journal of Food Engineering, Amsterdam, v.97, p.384-392, 2010.

ARVANITOYANNS, I. S.;VARZAKAS., T.H. Fruit/Fruit juice waste management: treatment methods and potential uses of treated waste. In: ARVANITOYANNIS, I. S. Waste Management for the food industries. Amsterdam: Academic Press, 2008. p. 579-628.

BUSTAMANTE, M. A.; M. D. PÉREZ-MURCIA; C. PAREDES; R. MORAL; A. PÉREZ-ESPINOSA; J. MORENO-CASELLES. Short-term carbon and nitrogen mineralisation in soil amended with winery and distillery organic wastes. Bioresource Technology, Amsterdam, v. 98, p. 3269-3277, 2007.

BUSTAMANTE, M. A.; MORAL, R.; PAREDES, C.; PÉREZ-ESPINOSA, A.; MORENO-CASELLES, J.; PÉREZ-MURCIA, M. D. Agrochemical characterisation of the solid by-products and residues from the winery and distillery industry. Waste Management, Amsterdam, v. 28, p. 372-380, 2008.
CORRALES, M.; TOEPFL, S.; BUTZ, P.D.; KNORR, B. TAUSCHER. Extraction of anthocyanins from grape by-products assisted by ultrasonics, high hydrostatic pressure or pulsed electric fields: A comparison. Innovative Food Science \& Emerging Technologies, Amsterdam, v. 9, n.1, p. 85-91. 2008.

\section{DOSSIÊ ANTIOXIDANTES. Food Ingredients} Brasil, São Paulo, n. 6, p. 16-30, 2009.

GEORGÉ, S.; BRAT, P.; ALTER, P.; AMIOT, M. J. Rapid determination of polyphenols and vitamin $\mathrm{C}$ in plant-derived products. Journal of Agricultural and Food Chemistry, Washington, v. 53, p.1.3701.373, 2005.

GIUSTI, M. M.; WROLSTAD, R. E. Characterization and measurement of anthocyanins by UV-visible spectroscopy. In: WROLSTAD, R. E. (Ed.). Current protocols in food analytical chemistry. New York: John Wile., 2001.

JU, Z. Y.; HOWARD, L. R. Subcritical water and sulfured water extraction of anthocyanins and other phenolics from dried red grape skin. Journal of Food Science, Chicago, v. 70. n.4, p. S270-S276, 2005.

KOSSEVA, M. R. Processing of food wastes. In: TAYLOR, S. Advances in food and Nutrition research. Burlington: Academic Press, 2009. v.58, p.57-136.

LEIFERT, W. A.; ABEYWARDENA, M. Y. Cardioprotective actions of grape polyphenols. Nutrition Research, New York, v. 28, n.11, p.729737,2008

MAZZA, G. Anthocyanins in grapes and grape products. Critical Reviews in Food Science and Nutrition, London, v. 35, n.4, p. 341-371, 1995.

MAZZA, G.; FUKUMOTO, L.; DELAQUIS, P.; GIRARD, B.; EWERT, B. Anthocyanins, Phenolics, and Color of Cabernet Franc, Merlot, and Pinot Noir Wines from British Columbia. Journal of Agricultural and Food Chemistry, Washington, v.47, p.4.009-4.017, 1999 . 
MELLO, L. M. R. Vitivinicultura brasileira: panorama 2011. Bento Gonçalves: Embrapa Uva e Vinho, 2012. 4p. (Comunicado Técnico, 115). Disponível em: <http://www.cnpuv.embrapa.br/ publica/comunicado/cot115.pdf $>$. Acesso em: 23 jul. 2012.

MONTEALEGRE, R.; RODRÍGUEZ; PECES, R. ROMERO; VOZMEDIANO, J. L. CHACÓN; GASCUEÑA, J. MARTINÉZ; ROMERO, E. GARCÍA. Phenolic compounds in skins and seeds of ten grape Vitis vinifera varieties grown in a warm climate. Journal of Food Composition and Analysis, Amsterdam, v.19, p. 687-693, 2006.

MUÑOZ, O.; SEPÚLVEDA, M.; SCHWARTZ, M. Effects of enzymatic treatment on anthocyanic pigments from grape skin from chilean wine. Food Chemistry, Kidlington, v. 87, n.4, p. 487-490, 2004.

NAZCK, M.,;SHAHIDHI, F. Extraction and analysis of phenolics in food. Journal of Chromatography A, Amsterdam, v. 1054, n.1/2, p. 95-111, 2004.

PALENZUELA, B.; ARCE, L.; MACHO, A.; MUÑOZ, E.; RIOS, A.; VALCARCEL, M. Bioguided extraction of polyphenols from grape marc by using an alternative supercritical-fluid extraction method based on a liquid solvent trap. Analytical and Bioanalytical Chemistry, Heidelberg, v. 378, n.8, p. 2021-2027, 2004.

PERESTRELO, R.; LU, Y.; SANTOS, S. A. O.; SILVESTRE, A. J. D.; NETO, C. P.; CÂMARA, J. S.;ROCHA, S. M. Phenolic profile of sercial and tinta negra Vitis vinifera L. grape skins by HPLCDAD-ESI-MSn Novel phenolic compounds in Vitis vinifera L. grape. Food Chemistry, Kidlington, v. 135, p. 94-104, 2012.

PETTI, S.; SCULLY, C. Polyphenols, oral health and disease: A review. Journal of Dentistry, Kidlington, v. 37, n.6, p. 413-423, 2009.

RATNASOORIYA, C. C.; RUPASINGHE, H. P. V. Extraction of Phenolic compounds from grapes and their pomace using beta-cyclodextrin. Food Chemistry, Kidlington, v. 134, n.2, p.625-631, 2012.
RE, R.; PELLEGRINI, N.; PROTEGGENTE, A.; PANNALA, A.; YANG, M.; RICE-EVANS, C. Antioxidant activity applying an improved ABTS radical cation decolorization assay. Free Radical Biology \& Medicine, New York, v.26, p. 12311237, 1999.

RUFINO, M. S. M.; ALVES, R. E.; DE BRITO, E. S., DE MORAIS, S. M.; SAMPAIO, C. G.; PÉREZ-JIMENEZ, J.; SAURA-CALIXTO, F. D. Metodologia científica: determinação da atividade antioxidante total em frutas pela captura do radical livre ABTS +. Fortaleza: Embrapa Agroindústria Tropical. 2007. 4 p. (Comunicado Técnico, 128). Disponível em: <http:/www.cnpat.embrapa.br/ cnpat/down/index.php?pub/Cot 128.pdf $>$. Acesso em: 25 ago. 2007.

SAntiago, M. C. P. A. Avaliação via Cromatografia Líquida de Alta Eficiência do efeito da microfiltração do suco da amora-preta (Rubus spp.) sobre a composição de suas antocianinas majoritárias. 2010. 92 f. Dissertação (Mestrado em Processos Químicos e Bioquímicos) - Escola de Química, Universidade Federal do Rio de Janeiro, Rio de Janeiro, 2010.

SHRIKHANDE, A. J. Wine by-products with health benefits. Food Research International, Kidlington, v. 33, p. 469-474, 2000.

SINGLETON, V. L.; ROSSI, J.A. Colorimetry of total phenolics with phosphomolybdic-phosphotungsticacid reagents. American Journal of Enology and Viticulture, Davis, v. 16, p. 144-168, 1965.

SPIGNO, G.; TRAMELLI, L.; DE FAVERO, D. M. Effects of extraction time, temperature and solvent on concentration and antioxidant activity of grape marc phenolics. Journal of Food Engineering, Amsterdam, v. 81, p. 200-208, 2007.

VALDUGA, E.; LIMA, L.; PRADO, R.; PADILHA, F. F.; TREICHEL, H. Extração, secagem por atomização e microencapsulamento de antocianinas do bagaço de uva "Isabel" (Vittis labrusca). Ciências e Agrotecnologia, Lavras, v. 32, n.5, p. 568-574, 2008 . 